\title{
L'ISCRIZIONE MEDIEVALE DI SANT'ELIA VECCHIO A CURINGA (CZ) E LA FONDAZIONE DEL MONASTERO
}

\author{
Adalberto Magnelli \\ Università di Firenze \\ adalberto.magnelli@unifi.it
}

\begin{abstract}
Da un riesame dell' iscrizione medievale di Curinga, emerge la possibilità che il monastero possa essere il monastero imperiale menzionato nell'atto di donazione di Roberto il Guiscardo del 1062 e quindi fondato nel periodo medio bizantino.

PAROlE CHIAVE: Curinga, monastero, iscrizione, data di fondazione, Calabria bizantina.
\end{abstract}

THE MEDIEVAL INSCRIPTION FROM SANT'ELIA VECCHIO IN CURINGA AND THE FOUNDATION OF THE MONASTERY

\section{ABSTRACT}

A re-examination of the medieval inscription found in Curinga, Southern Italy, reveals the possibility that the monastery there was the "imperiale monasterium" mentioned in the donation deed of 1062 by Robert Guiscard and therefore it was founded in the Middle Byzantine period.

KeYwOrDs: Curinga, Monastery, Inscription, foundation date, Byzantine Calabria

L'eremo o monastero di Sant'Elia Vecchio' ${ }^{1}$, immerso entro un paesaggio bucolico e affascinante, a $400 \mathrm{~m}$ s.l.m. in località Corda di Curinga $(\mathrm{Cz})$, è di certo uno dei monumenti più misteriosi nell'ambito delle ricerche e degli studi sulla Calabria di età medievale.

Notizie su questo complesso sono quasi del tutto assenti. A eccezione di documenti tardi (XVII secolo) $)^{2}$, che lo menzionano come ormai cenobio carmelitano, non esistono dati certi sull'epoca della sua fondazione e sulle vicende che lo hanno interessato nell'età precedente. Dall'analisi strutturale eseguita sulle murature del complesso monastico ${ }^{3}$, sembra sia possibile tuttavia riconoscere almeno cinque distinte fasi di vita, la più antica delle quali risalirebbe presumibilmente a epoca pre-normanna ${ }^{4}$.

Unico elemento che, in qualche modo, potrebbe risultare utile ai fini di una più precisa contestualizzazione cronologica, è costituito da un'iscrizione dipinta su 


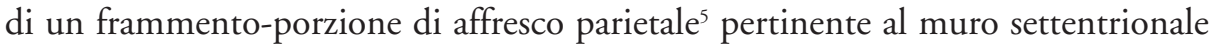
della cappella di Sant'Elia ${ }^{6}$, contigua all'edificio ecclesiastico del monastero.

L'editio princeps del testo, a cura di Franco Mosino ${ }^{7}$, è la seguente:

5.

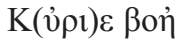

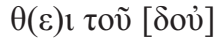

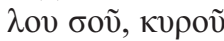

$$
\begin{aligned}
& \operatorname{Ko}(v) \sigma \tau \alpha(v \tau i v v v) \dot{\varepsilon} \pi(1 \sigma)- \\
& \text { ко̉สov } \\
& \lambda \alpha c---
\end{aligned}
$$

Il testo, di non semplice lettura, prosegue oltre la linea 6 con altri segni, indicati nell'apografo riportato dall'editore ${ }^{8}$, che tuttavia dispera di poter interpretare. Secondo la tipologia dei caratteri, si tratterebbe di un'epigrafe della fine del XIV o, al massimo, dei primissimi anni del secolo successivo. Si ha menzione di un certo vescovo Costantino, probabile donatore o eventuale sponsor dell'erezione della cappella entro il complesso del monastero.

L'unicità del documento e la particolarità del suo rinvenimento - si trovava ancora in situ all'epoca dell'edizione - ci inducono a riflettere nuovamente sul testo per verificare la possibilità di estrarre altre informazioni, che risulterebbero oltremodo

${ }^{1}$ La denominazione è utilizzata per distinguere il complesso monastico dall'analogo Sant'Elia, denominato "nuovo", ove si trasferirono i monaci Carmelitani nella seconda metà del XVII secolo.

${ }^{2}$ La prima attestazione documentaria sul complesso risulterebbe la bolla di Papa Alessandro VI Borgia del 31 maggio 1493, nella quale il monastero è definito basiliano: in proposito vd. il Regesto Vaticano in Russo, 1975; Parisi, 1987: X-XI e Augruso, 2007: 41. Sulla restante documentazione seicentesca in generale vd. Boaga, 1995.

${ }^{3}$ Gli scavi sono stati condotti nel 1991: vd. Cuteri, 1993: 556. Per l'individuazione delle fasi del complesso monastico sulla base di una dettagliata analisi di "stratigrafia muraria": vd. Donato, 1998: 173-183.

${ }^{4}$ Donato, 1998: 175-177. Le decorazioni architettoniche più antiche e la tipologia dell'edificio ecclesiastico potrebbero far pensare a una datazione fra IX e XI secolo per le fasi iniziali: Donato, 1998: 179-181; Donato, 1999: 365-379. Contrariamente Cuteri, 2009: 34-36.

${ }^{5}$ I pochi tratti dipinti riconoscibili rimanderebbero a caratteri tipici della “... pittura murale bizantina provinciale”, diffusa in Calabria in ambito basiliano (IX-XI sec.): cfr. Donato, 1998: 181. Sulla pittura ad affresco in Italia meridionale vd. la messa a punto in Falla Castelfranchi, 1991; Pace, 2012 (ivi bibliografia).

${ }^{6}$ Vi si accede direttamente dalla navata della chiesa: Donato, 1998: 171. Sulla tipologia funzionale e architettonica delle "cappelle" annesse alle chiese monastiche interessanti osservazioni in Babić, 1969; Ćurčić, 1977: 98-108; Popović, 2007: spec. 50 ss.; Gerstel, 2015: 6-32. Un esempio simile, con nicchie sul fondo e affresco, è rintracciabile nella cappella di Sant'Atanasio a Didymoteicho per cui vd. Ousterhout, 1999: spec. Tavv. a 204-205. Durante le fasi di scavo si ebbe il rinvenimento «... all'interno della cappella dedicata a Sant'Elia, di un'insolita sepoltura scavata nel robusto pavimento di malta»: Cuteri, 2009: 33.

${ }^{7}$ Mosino, 1996: 105-106.

${ }^{8}$ Mosino, 1996: 105. 

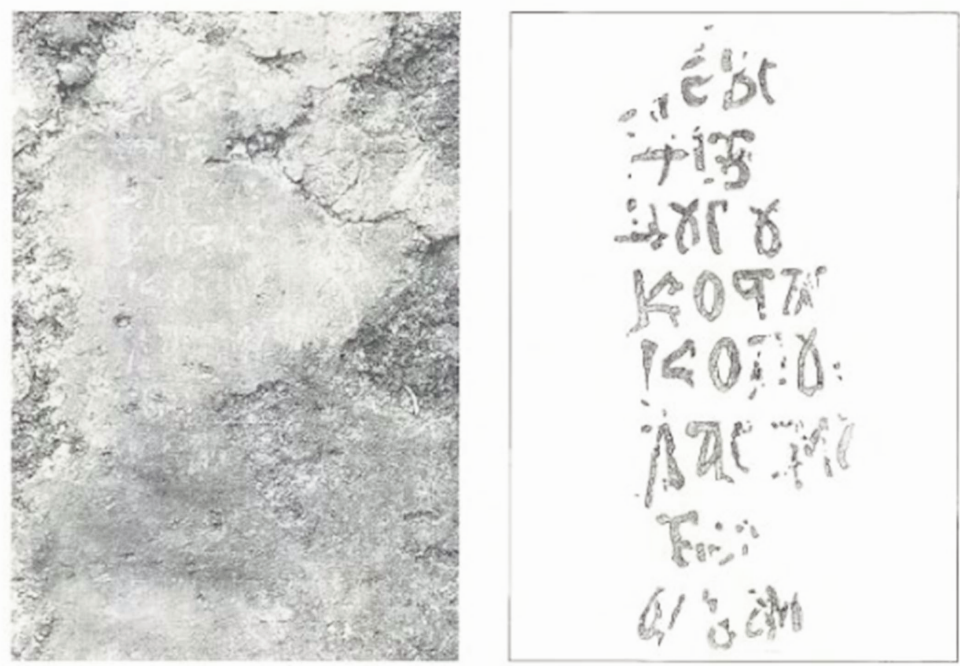

Figura 1. Iscrizione (a sin.) greco-bizantina presso Sant'Elia Vecchio di Curinga e (a destra) apografo della medesima (licenza wikimedia commons).

importanti per definire meglio l'intera questione della cronologia iniziale del monumento calabrese.

Le considerazioni che seguono sono il frutto di una rilettura condotta sull'apografo e l'immagine presentata in $e d$. $p r$. (figura 1) oltreché di una successiva reinterpretazione del testo in connessione con altra documentazione riconducibile all'esistenza del Sant'Elia Vecchio in epoca alto medievale?.

Riesaminando le prime linee dell'iscrizione non sfugge la difficoltà di intravedere a linea 3 la sequenza кupoṽ, indicata come leggibile e certa in ed. pr. Osservando il ductus dei caratteri e l'impaginato, che probabilmente prevedeva per il testo "un ruolo" didascalico di accompagnamento ${ }^{10}$ a un'immagine di una figura della quale si conservano tracce esigue sull'intonaco immediatamente a destra ${ }^{11}$, pare

'Si fa qui riferimento al famoso documento di donazione, da parte del duca Roberto il Guiscardo, della rifondata abbazia di Santa Maria di Sant'Eufemia Vetere ai monaci benedettini, sul quale vd. infra nel testo.

${ }^{10}$ Cuteri, 2009: 39 afferma in proposito che «... il riquadro iscritto (corsivo d.s.) ricorda simili cartigli presenti in altri opere pittoriche bizantine o di tradizione bizantina in Calabria». Per l'impaginato, con i caratteristici compendi tachigrafici, si vd. ad esempio l'immagine dello iereus Nicola Kakomerotos nella chiesa di San Michele Arcangelo Polemita, nel Mani Peloponnisou: sul quale adesso Gerstel, 2015: 129-130, fig. nr. 95.

${ }^{11}$ Donato, 1998: 172: «... Sul muro nord si trovano le tracce di un affresco (USM 84) raffigurante un santo o un monaco, con i resti di un'epigrafe in greco ...». La presenza di monaci su 
possibile ipotizzare la presenza al massimo di 6 lettere per linea, con un minimo forse di 4; il che dovrebbe far escludere automaticamente la presenza del termine proposto. Allo stesso modo, nella linea successiva, dopo le prime lettere, pertinenti al nome di Costantino, difficilmente gli altri caratteri sembrerebbero presentare le forme di $\mathrm{E}$ o П. Lo stesso apografo, che accompagna la riproduzione fotografi$\mathrm{ca}$, sembra escludere tale evenienza, presentando segni maggiormente riconducibili a una lettera triangolare, seguita da un'asta verticale. Escluderei quindi la menzione

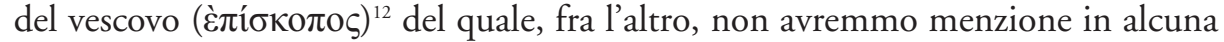
cronotassi locale relativa alla fine del XIV secolo.

A l. 6 l'editore legge la sequenza $\Lambda \mathrm{AC}$; se la seconda e terza lettera in qualche modo possono rendere ragione di tale interpretazione, un confronto con la parte inferiore di beta presente a 1.1 e con altre analoghe tipologie grafiche di XII-XIV seco$1 \mathrm{o}^{13}$, indurrebbero a preferire una lettura $\beta \alpha \mathrm{c}$, indicando il sigma finale secondo la consueta caratterizzazione lunata ad apici ${ }^{14}$.

La susseguente rilettura di quanto segue dopo 1. 6, pur con le dovute riserve imputabili allo stato di non perfetta conservazione dell'intonaco e considerando lo stile tachigrafico e le abbreviazioni tipiche dello stile, potrebbe far propendere per la seguente ipotetica restituzione nella quale si indicano in neretto le nuove proposte:

5.

$$
\begin{aligned}
& \mathrm{K}(\dot{v} \rho \mathrm{l}) \mathcal{\varepsilon}^{15} \beta \text { ßó }
\end{aligned}
$$

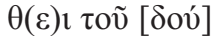

$$
\begin{aligned}
& \lambda \text { ov } \sigma 0 \tilde{v}^{16} \\
& \operatorname{Ko}(v) \sigma \tau(\alpha v \tau \tilde{\imath} v o v)^{17}, \delta \mathbf{\delta}(\alpha)-
\end{aligned}
$$

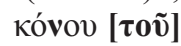

rappresentazioni parietali è tipica anche dei monasteri rupestri di Cappadocia, altra zona provinciale spesso messa in relazione con l'area italica meridionale, si cfr. ad es. la raffigurazione del monaco Macario nella chiesa dell'eremitaggio di San Giovanni con l'iscrizione 'Y

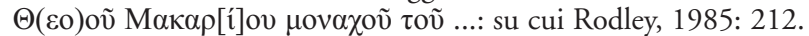

${ }^{12}$ La terza lettera di 1.5 , a ben vedere, non è inequivocabilmente da leggersi $\Pi$, bensì $\mathrm{N}$ come è possibile evincere dal tratto obliquo leggermente ondulato e discendente in alto a sinistra (cfr. fig. 1, riproduzione fotografica). Per simile tipologia di N vd. Moutsopoulos, 1990: 99, pl. 25, nrr. 1-2.

${ }_{13} \mathrm{Si}$ prendano a riferimento nel caso specifico le tavole paleografiche riportate in Moutsopoulos, 1990: $79 \mathrm{pl}$. 5, specialmente nrr. 18, 20 e 22. Nel tracciare l'evoluzione dei caratteri, fra XIII e XV secolo, a proposito della lettera B lo stesso autore afferma (ivi: 60): «... Parfois, la parte inférieure s'elargit en devenant triangulaire».

${ }^{14}$ Moutsopoulos, 1990: 87, pl. 13.

${ }^{15}$ Mancando la porzione di intonaco immediatamente superiore a quella che conserva la presunta prima linea del testo, non potremmo escludere anche un rigo precedente con la sequenza $\theta \varepsilon 0 \tau 0-$ che si legherebbe alle lettere successive a formare il nome della Vergine Theotokos ( $\Theta \varepsilon o \tau o ́ \mid \kappa \varepsilon)$, riferimento frequente nelle invocazioni.

${ }^{16}$ L'uso del genitivo nell'invocazione è meno diffuso rispetto alla forma regolare in dativo, ma ben presente nelle iscrizioni bizantine dell'Italia meridionale, cfr. exempli gratia Guillou, 1996: 193-194, nr. 183 da Vasto (dataz. 1054).

${ }^{17}$ L'abbreviazione $\operatorname{Ko}(v) \sigma \tau$ è ampiamente diffusa nell' usum epigrafico paleocristiano e medievale in genere. 


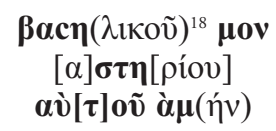

La tachigrafia, caratteristica del dittongo ov e dell'articolo genitivo maschile

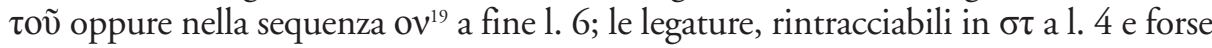
$\sigma \tau \eta$ a l. 7; le abbreviazioni presenti a ll. 4, 6 e 8, fanno dunque propendere per un testo raccolto entro uno spazio non troppo dilatato, con ratio di 4-6 lettere per linea di scrittura. Possiamo tradurre:

Signore, proteggi il Tuo servo Costantino, diacono ${ }^{20}$ di questo monastero imperiale, amen.

Si tratta di una normale invocazione, forse riferita a un personaggio, coinvolto nella stessa edificazione della cappella di Sant ${ }^{3} \operatorname{Elia}^{21}$. Il nuovo e interessante dato che risulterebbe nel caso in cui fosse possibile confermare l'ipotesi di lettura qui proposta, risiederebbe senza dubbio nel titolo di "imperiale" che accompagna la menzione dell'edificio monastico stesso. Conosciamo infatti un diploma normanno, attribuito a Roberto il Guiscardo e datato $1062^{22}$, che, in seguito alla rifondazione o restauro del monastero di Santa Maria di sant'Eufemia, nel territorio di Nicastro, non troppo distante da Curinga, attribuisce alle proprietà del nuovo istituto quelle del "monastero imperiale di Sant'Elia" ${ }^{23}$. Tradizionalmente si è riconosciuto in quest'ultima menzione il riferimento al monastero di Sant'Elia a Palmi, fondato da S. Elia da Enna nel X secolo, riccamente dotato dal basileus Leone VI e ben noto da altre fonti storiche $\operatorname{coeve}^{24}$. Pur tuttavia, la relativa distanza delle due fondazioni e la presenza nel testo

${ }^{18}$ L'abbreviazione è abbastanza comune nella sigillografia.

${ }^{19}$ Le lettere sono sovrapposte con omicron in basso e ny in apice, così come nell'es. riportato da Moutsopoulos, 1990: 94, pl. 20,3.

${ }^{20}$ Probabilmente il personaggio svolgeva la duplice funzione di diacono e monaco, ovvero si tratta di uno ierodiacono: cfr. Patrologia Latina LXVI, coll. 65-66 (Epistulae di papa Agapito). Per la regola concernente l'ammissione di diaconi nei monasteri dell'Italia meridionale e Sicilia a partire dal XII secolo, si cfr. il typikon di San Salvatore a Messina per cui adesso Torre, 2017: 59 ss. Sulla figura di presbiteri, monaci o diaconi come offerenti in affreschi bizantini di XIV secolo nel territorio del Salento vd. Safran, 2014: 65-70.

${ }^{21}$ Cuteri, 2009: 39. Per simili invocazioni in testi di dedica cfr. Kalopissi Verti, 1992: passim. Su raffigurazioni murali e ritratti in genere di donatori all'interno di edifici ecclesiastici bizantini vd. ora Franses, 2018.

${ }^{22}$ Il diploma, originariamente in greco, è noto attraverso copie latine risalenti al XVI e XVII secolo. Sulla vexata quaestio della autenticità di simile documento vd. Parisi, 1952: 667-672; Ménager, 1959: 4-22; Laurent, 1960: 59-62; Pontieri, 1964: 288-290; Parisi, 1965: 137-143; Scordovillo, 1972: 13-14; Miceli di Serradifalco, 1977: 241-260; Burgarella, 1999: 389 e s.

${ }^{23}$ Il testo latino riporta: Dedi quoque Imperiale Monasterium Sancti Eliae cum villanis et omnibus pertinentibus et appendicibus suis ... per le redazioni che si susseguono nelle varie copie vd. Ménager, 1959: 4 ss.

${ }^{24}$ Su quest'ultima identificazione Parisi, 1987; Burgarella, 1999: 391. 
di Curinga del titolo $\beta \alpha \sigma \imath \lambda \iota \kappa \delta^{25}$ riferito al cenobio, potrebbero far riconsiderare nuovamente tale identificazione. Nella buona sostanza il monastero citato nel diploma del Guiscardo potrebbe più facilmente essere quello di Curinga, in località congruente con gli altri possedimenti di Sant'Eufemia e dunque più facilmente controllabile dall'abate ${ }^{26}$; d'altra parte la menzione in un diploma della metà dell'XI secolo costituirebbe un terminus ante quem per la fondazione dell'eremo stesso, confermando un possibile legame con il basileus costantinopolitano che, proprio in età medio bizantina, aveva favorito e patrocinato la nascita di più edifici monastici nel tema di Calabria $^{27}$. Il dato epigrafico andrebbe quindi a confermare quanto emerso dalle indagini sulle strutture murarie e indurrebbe a considerare il periodo fra X e XI secolo come il più probabile per la nascita della comunità monastica nel territorio di Curinga.

RECIBIDO: enero 2021; ACEPTADO: abril 2021.

\section{BIBLIOGRAFIA}

Augruso, S. (2007): Memoria Ecclesiae memoria populi. Vita religiosa e vita civile di una comunità calabrese, Qualecultura ediz., Vibo Valentia.

BABIĆ, G. (1969): Les chapelles annexes des églises Byzantines. Fonction liturgique et programmes iconographiques, Bibliothèque des Cahiers Archéologiques, III, C. Klincksieck, Paris.

BOAGA, E. (1995): «La presenza dei carmelitani in Calabria e il convento di S. Elia in Curinga», Carmelus 42/1: 197-236.

Burgarella, F. (1999): «A proposito del diploma di Roberto il Guiscardo per l'abbazia di Santa Maria di Sant'Eufemia (1062)», in G. De Sensi Sestito (a cura di), Tra l'Amato e il Savuto, II, Studi sul lamentino antico e tardo-antico, Rubbettino, Soveria Mannelli (Catanzaro), pp. 381-406.

ĆURČIĆ, S. (1977): «Architectural Significance of Subsidiary Chapels in Middle Byzantine Churches», JSAH 36: 94-110.

CUTERI, F. A. (1993): «(CZ) Curinga, Eremo di S. Elia Vecchio. 1991, aggiornamento schede 1971-1991», Archeologia Medievale 20: 556.

Cuteri, F. A. (2009): «Come sul Monte Carmelo. L'Eremo di Sant'Elia Vecchio a Curinga», Esperide 3-4: 33-40.

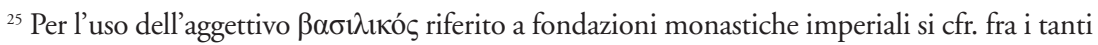

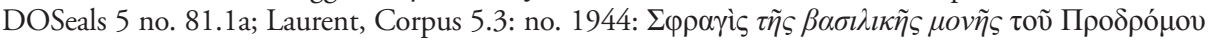

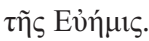

${ }^{26}$ Si osservi come alla fine del medioevo il cenobio di Sant'Elia Vecchio rientri significativamente proprio nella giurisdizione del baliaggio di S. Eufemia: vd. Cuteri, 2009: 36.

${ }_{27}^{27}$ Sul monachesimo calabrese di rito greco in età pre-normanna vd. da ultimo Roma, 2017: 359-372. Un interessante caso di patrocinio imperiale riguarda la fondazione calabrese di Santa Maria del Patir di Rossano voluta da San Bartolomeo Simeri agli inizi del secolo XII. Sui rapporti fra le comunità monastiche di Calabria e le fondazioni imperiali del Monte Athos fra X e XI secolo vd. Morini, 2018: 191-207. 
DonATO, E. (1998): «Archeologia dell'architettura in Calabria: il monastero di S. Elia Vecchio a Curinga (CZ)», Archeologia dell'architettura 3: 169-183.

Donato, E. (1999): «Le fasi più antiche del monastero di S. Elia Vecchio a Curinga», in G. DE SensI SESTITO (a cura di), Tra l'Amato e il Savuto, II, Studi sul lamentino antico e tardo-antico, Rubbettino, Soveria Mannelli (Catanzaro),pp. 365-379.

Falla CastelfranchI, M. (1991): «Disiecta membra. La pittura bizantina in Calabria (sec. X-XIV)», in AA.VV. (a cura di), Calabria bizantina: testimonianze d'arte e strutture di territori, Atti dell'VIII e IX Incontro di studi bizantini, Reggio Calabria, Vibo Valentia, Tropea 17-19 maggio 1985 e 16-18 dicembre 1988, Rubbettino, Soveria Mannelli (Catanzaro), pp. 21-61.

FRANSES, R. (2018): Donor portraits in Byzantine Art: the vicissitudes of contact between human and divine, Cambridge University Press, New York.

Gerstel, S. J. (2015): Rural lives and landscapes in late Byzantium: Art, Archaeology and Ethnography, Cambridge University Press, New York.

Guillou, A. (1996): Recueil des inscriptions grecques médiévales d'Italie, Collection de l'École Française de Rome, 222, École Française de Rome, Rome.

KALOPISSI VERTI, S. (1992): Dedicatory inscriptions and Donor portraits in thirteenth-century churches of Greece, Österreichische Akademie der Wissenschaften, Veröffentlichungen der Kommission für die Tabula Imperii Byzantini, Band 5, Verlag der Österreichischen Akademie der Wissenschaften, Wien.

Laurent, H. M. (1960): «L'abbazia di Sant'Eufemia e il Vespro Siciliano», Calabria nobilissima 15: 59-62.

MéNAGER, L. R. (1959): «Les fondations monastiques de Robert Guiscart, Duc de Pouille et de Calabre», Quellen und Forschungen aus italienischen Archivien und Bibliotheken 39: 1-116.

Miceli di Serradifalco, A. (1977): «Lordine di S. Giovanni di Gerusalemme in Calabria dal XII al XV secolo", Studi meridionali 10: 241-260.

Morini, E. (2018): «Monastic interactions between Calabria and Mount Athos in the Middle Ages», in B. Crostini - I. A. MURZAKU (eds.), Greek Monasticism in southern Italy: the Life of Neilos in context, Routledge, New York: 191-207.

Mosino, F. (1996): «Iscrizione greca medievale da Curinga (Catanzaro)», Xenia Antiqua 5: 105-106.

Moutsopoulos, N. K. (1990): «La morphologie des inscriptions byzantines et post-byzantines de Grèce», Cyrillomethodianum 3: 53-105.

Ousterhout, R.G. (1999): «A Byzantine Chapel at Didymoteicho and its Frescoes», in A. IACOBINI M. Della Valle, L’arte di Bisanzio e l'Italia al tempo dei Paleologi, 1261-1453, Argos ediz., Roma, pp. 195-207.

PACE, V. (2012): «La transperiferia bizantina nell'Italia meridionale del XIII secolo. Affreschi in chiese del Salento pugliese, della Basilicata e della Calabria», in F. JouberT - J.-P. CAILlet, Orient et Occident méditerranéens au XIII siècle - Les programmes picturaux, Actes du colloque international organisé à l'Ecole française d'Athènes les 2-4 avril 2009, Picard, Paris, pp. 215-234.

PARISI, A. F. (1952): «Su un pretesto diploma di Ruggero conte di Sicilia al monastero benedettino di S. Eufemia in Calabria», Nova Historia 3: 667-672.

PARISI, A. F. (1965): «L'elezione dell'abate nei monasteri benedettini pinerolesi e calabresi nei secc. XI e XII", Historica 18: 132-143.

PARISI, A. F. (1987): Lacconia: un antico insediamento, Laruffa ediz., Reggio Calabria.

PONTIERI, E. (1964): Tra i Normanni nell'Italia Meridionale, Edizioni Scientifiche Italiane, Napoli ( $2^{\circ}$ ediz.). 
Popović, S. (2007): «Dividing the indivisible: The monastery space - secular and sacred», Zbornik radova Vizantoloskog institute 44: 47-65.

Rodley, L. (1985): Cave Monasteries of Byzantine Cappadocia, Cambridge University Press, New York.

Roma, G. (2017): «Il fenomeno monastico sul territorio dell'attuale Calabria dalle origini alla conquista dei Normanni», in C. Masseria - E. Marroni (a cura di), Dialogando. Studi in onore di Mario Torelli, ETS edizioni, Pisa, pp. 359-372.

Russo, F. (1975): Regesto vaticano per la Calabria, G. Gesualdi, Roma.

SAFRAN, L. (2014): The medieval Salento: Art and Identity in Southern Italy, University of Pennsylvania Press, Philadelphia.

SCORDOVILLO, F. (1972): «II baliaggio di S. Eufemia del Golfo di proprietà dei cavalieri Gerosolomitani», Brutium 51/2: 13-14.

Torre, C. (2017): «I Typika monastici Italo-Greci», in B. CrostinI - I. A. MurZAKU (eds.), Greek Monasticism in southern Italy: the Life of Neilos in context, Routledge, New York, pp. 44-77. 